\title{
IMAGING WITH A HOMOGENEOUS MILLIMETER ARRAY
}

\author{
M.A. HOLDAWAY \\ NRAO, PO Box O, Socorro, NM 87801
}

\begin{abstract}
Presented here are the results of simulations of the effects of various errors on the imaging capabilities of the compact $(72 \mathrm{~m})$ configuration of a 39 element homogeneous millimeter array using the mosaic algorithm to image sources larger than the primary beam. Included in the errors investigated to date are systematic and random pointing errors, Gaussian receiver and atmospheric noise, and primary beam errors. Various methods of quantifying the quality of the reconstructed image are also discussed.
\end{abstract}

\section{INTRODUCTION}

The proposed Millimeter Array (MMA) will image many objects that require both interferometric measurements for high resolution and total power measurements to recover all the flux (see Brown, these proceedings). Conventional wisdom dictates that the total power measurements be performed with a single dish 2-3 times as large as the shortest measured interferometer spacings. Recently, it has been demonstrated that excellent mosaic images can be reconstructed from an array which measures interferometric spacings and total power with the same antennas (the homogeneous array concept; see Uson and Cornwell, these proceedings). For details of the mosaic algorithm, see Cornwell (1988).

This work studies the effects of noise, pointing errors and primary beam errors on the quality of the mosaic images generated from a homogeneous array of 397.5 meter elements in a compact configuration with a maximum baseline of 72 meters, and explores the implications for the MMA design.

\section{EVALUATING RECONSTRUCTED IMAGE QUALITY}

Knowing the model brightness distribution permits sophisticated fidelity measures to be employed:

- the image dynamic range (DR) given by peak over off-source rms. - the image fidelity index (FI), given by the on-source median of the reconstructed image divided by the absolute value of the difference between the reconstructed image and the model brightness distribution. The FI is the inverse of the typical on-source fractional error. 
- the residual visibility ( $\mathbf{R V}$ ) plot. The RV plot is constructed by differencing the reconstructed image and the convolved model, Fourier transforming, averaging in radial bins, and normalizing by the binned, FT of the model. The RV plot can answer the question: "How good is the image reconstruction on the $5-10 \mathrm{~m}$ spacings where the total power and interferometer sensitivity patterns meet?"

It should be emphasized that none of these methods can be used as an absolute measure of image fidelity: they should be used as relative measures. To calibrate our understanding of these fidelity measures, we compare simulated error-free VLA and MMA images. A processed optical image of an HII region in M31, scaled to $100^{\prime \prime}$, was used as the model brightness distribution. Perfect $(u, v)$ data were produced for the VLA B and D arrays (25 minutes each) at $1.5 \mathrm{GHz}$, yielding a 3.'6 beam. CLEAN deconvolution of the VLA data yields $\mathrm{DR}=1240$ and $\mathrm{FI}=10 . \mathrm{MEM}$ deconvolution yields $\mathrm{DR}=840$ and $\mathrm{FI}=14$. At $230 \mathrm{GHz}$, the $72 \mathrm{~m}$ configuration of the MMA has a $3{ }^{\prime \prime} 6$ beam and the model was covered by 49 fields separated by $17^{\prime \prime}$, each with 1 minute integrations. The MMA image has DR $=3160$ and FI $=150$. Departures from the model are due to deconvolution errors only. The low FI indicates on-source deconvolution errors are $\sim 1$ order of magnitude worse than off-source errors. The RV plots for the VLA and the MMA images are compared in Fig. 1. The MMA is designed to measure all spatial frequencies out to some maximum value, and the MMA's RV plot reflects this. The quality of the VLA images indicates good science can be done with low fidelity images.

Of the above methods, only the DR can be measured in real observations. (However, see Biretta, these proceedings, for a cousin of the FI applied to two VLBI observations on consecutive days.) Since no one knows what the RV plot or the FI is for actual observations, these methods can only be used for evaluating simulation results.

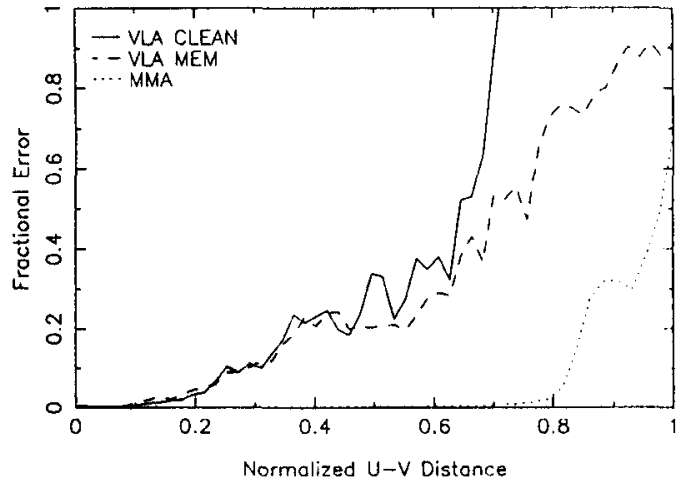

Fig. 1. Residual visibility plots for error-free VLA and MMA simulations.

\section{SIMULATION RESULTS}

\section{Primary Beam Errors}

Since the mosaic algorithm images objects much larger than the antenna primary beam, errors in the primary beam model will limit the fidelity of the 
reconstructed image. We assumed perfect agreement down to some fraction of the beam peak below which the actual beam was given as a Gaussian while the model beam was the diffraction response. With errors as high as the $7 \%$ level the reconstructed images still possessed DR of 3000:1 and FI of 140. Good images can be produced with higher errors, but MEM converges very slowly.

\section{Antenna Pointing Errors}

Our pointing error model includes constant and time varying systematic and purely random pointing errors in azimuth and elevation (Holdaway, 1990). The largest reconstruction errors occur between $2 \mathrm{~m}$ and $12 \mathrm{~m}$. In the image plane, the errors are characterized by large, low brightness plateaus and holes about the true source emission. DR is plotted as a function of rms pointing error in Fig. 2, indicating DR of 750 can be achieved with 1.2 pointing errors. The FI, plotted against rms pointing error in Fig. 3, is about 20 for 1"'2 pointing errors and falls to about 10 for 2.3 pointing errors.

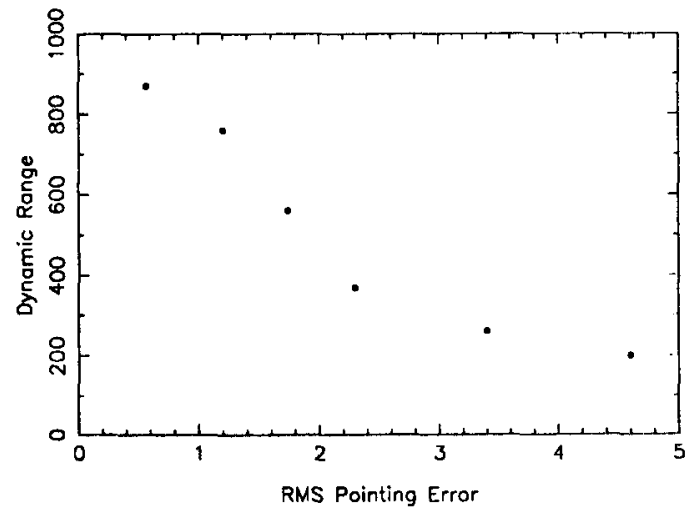

Fig. 2. Image DR as a function of rms pointing error for mosaiced MMA images.

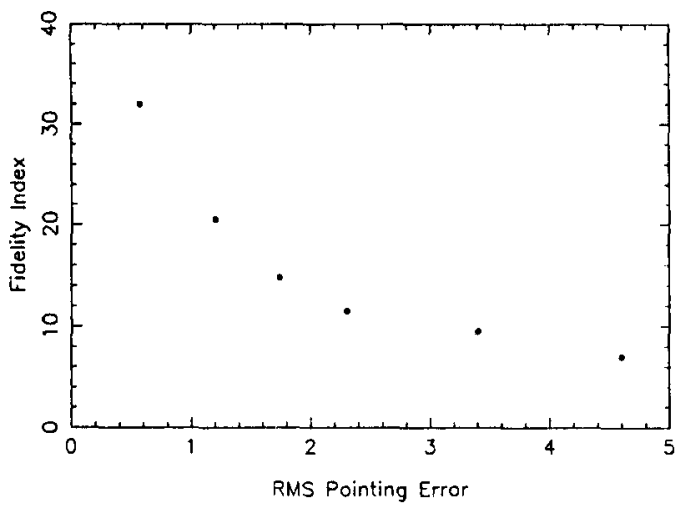

Fig. 3. Image FI as a function of rms pointing error for mosaiced MMA images. 


\section{Gaussian Noise and Pointing Errors}

Noise appropriate to $T_{s y s}=200 \mathrm{~K}, 1 \mathrm{MHz}$ bandwidth, 1 minute integration per field was added to simulated visibilities generated from the model scaled in brightness. Without pointing errors, DR increases linearly with source brightness temperature and FI increases with a slight roll off at high brightness temperatures as deconvolution errors become more important.

When 1".2 pointing errors are added in concert with Gaussian noise, DR increases similarly to the noise-only case but is limited by pointing errors at high brightness temperatures $(>50 K)$. The FI saturates at about 20 for brightness temperatures above $10 \mathrm{~K}$. By scanning over the source several times, some of the effects of the pointing errors will average down, and the FI increases almost to its value with no pointing errors. This improvement is highly dependent upon the pointing error model, the hour angles of observation, and the declination of the source. However, between multiple scans and calibration of a global component of the pointing errors, FI of around 60 can be expected for $T_{b}=40 \mathrm{~K}$.

\section{CONCLUSIONS}

The homogeneous array design of the proposed MMA is shown to be robust with respect to all errors we have simulated so far. Important design specifications that have been determined or reinforced by these simulations include:

- good knowledge and uniformity of the antenna primary beams down to the $7 \%$ level.

- pointing accuracy on the order of $1^{\prime \prime}$ rms.

- fast on-line control system and short set-up times (a few seconds) to allow for efficient short scans ( 5 seconds).

The extensive simulation campaign required by the MMA is helping to raise our consciousness of image fidelity. Perhaps simulations should be a routine part of imaging to give a realistic level of reconstruction errors and test the reality of uncertain features in reconstructed images.

\section{ACKNOWLEDGEMENTS}

Special thanks to Tim Cornwell and Juan Uson for the many discussions which have aided this work and helped me develop insight into the workings of the mosaic algorithms and the proposed MMA. Also, thanks to Darrel Emerson for challenging these results and requiring me to refine them.

\section{REFERENCES}

Cornwell, T.J., (1988), Astr. Ap., . 202, 316-321.

Cornwell, T.J., Holdaway, M.A., and Uson, J.M., in preparation.

Holdaway, M.A., (1990), Millimeter Array Memo 61, NRAO. 